\title{
Interactive effects of species richness and species traits on functional diversity and redundancy
}

\author{
Fredrik Dalerum • Elissa Z. Cameron - Kyran Kunkel • \\ Michael J. Somers
}

Received: 5 April 2010 / Accepted: 3 November 2010

(C) Springer Science+Business Media B.V. 2010

\begin{abstract}
The importance of species diversity for ecosystem function has emerged as a key question for conservation biology. Recently, there has been a shift from examining the role of species richness in isolation towards understanding how species interact to effect ecosystem function. Here, we briefly review theoretical predictions regarding species contributions to functional diversity and redundancy and further use simulated data to test combined effects of species richness, number of functional traits, and species differences within these traits on unique species
\end{abstract}

F. Dalerum $(\bowtie) \cdot$ M. J. Somers

Centre for Wildlife Management, Hatfield Experimental Farm, University of Pretoria,

0002, Pretoria, South Africa

e-mail: fredrik.dalerum@zoology.up.ac.za

M. J. Somers

e-mail: mjs@up.ac.za

F. Dalerum • E. Z. Cameron · M. J. Somers

Mammal Research Institute, Department of Zoology and

Entomology, University of Pretoria,

0002, Pretoria, South Africa

M. J. Somers

Centre for Invasion Biology, University of Pretoria,

0002, Pretoria, South Africa

K. Kunkel

WWF,

Bozeman, MT, USA

e-mail: kunkel@montana.net

Present Address:

E. Z. Cameron

School of Zoology, University of Tasmania,

Hobart, Australia

e-mail: ezcameron@utas.edu.au contributions to functional diversity and redundancy, as well as on the overall functional diversity and redundancy within species assemblages. Our results highlighted that species richness and species functional attributes interact in their effects on functional diversity. Moreover, our simulations suggested that functional differences among species have limited effects on the proportion of redundancy of species contributions as well as on the overall redundancy within species assemblages, but that redundancy rather was determined by number of traits and species richness. Our simulations finally indicated scale dependence in the relative effects of species richness and functional attributes, which suggest that the relative influence of these factors may affect individual contributions differently compared to the overall ecosystem function of species assemblages. We suggest that studies on the relationship between biological diversity and ecosystem function will benefit from focusing on multiple processes and ecological interactions, and that the relative functional attributes of species will have pivotal roles for the ecosystem function of a given species assembly.

Keywords Ecosystem function - Ecosystem services . Community ecology · Biodiversity . Theoretical ecology . Simulation experiments $\cdot$ Functional traits

\section{Introduction}

The importance of biological diversity for ecosystem function has emerged as a key question for conservation biology (Naeem et al. 1994; Schwarz et al. 2000; Hector et al. 2001; Loreau et al. 2001; Naeem 2002; Srivistava and Vellend 2005). While early work contrasted species richness to specific components of ecosystem function 
(reviewed in Balvanera et al. 2006), there has been a recent thrust towards understanding how species interact in their effects on ecosystem functioning and how these interactions are affected by different attributes among species (Reiss et al. 2009; Caliman et al. 2010). It is now generally accepted that the structure of species assemblages may be as important as their species richness (Naeem and Wright 2003; Petchey and Gaston 2006; Petchey et al. 2007; Griffin et al. 2009). This realization has underpinned the shift in focus of biodiversity research from species to functional diversity (i.e., "the value and range of those species and organismal traits that influence ecosystem functioning in a given system" Tilman 2001), with a subsequent increase in quantifications of functional diversity in empirical species assemblages (Fukami et al. 2005; Heino 2005; Micheli and Halpern 2005; Petchey et al. 2007; Bracken et al. 2009; Dalerum et al. 2009).

In parallel with the increased interest in functional diversity, there has been an intense debate regarding ecological redundancy, the concept that some species may not be required for ecosystem functioning, and its potential importance for ecosystem dynamics (Walker 1992; Lawton and Brown 1993; Walker 1995; Johnson et al. 1996; Loreau 2004). Although there is empirical evidence for ecological redundancy within fungal and invertebrate communities (Dang et al. 2005; McKie et al. 2008), species richness has in other communities been directly related to functional diversity (Micheli and Halpern 2005; Petchey et al. 2007), indicating no or very low redundancy in these communities. Holling (1973) introduced the concept of ecosystem resilience as a way to improve our understanding of nonlinear ecological processes. Such ecological resilience can be regarded as a measure of how much disturbance an ecosystem can absorb without changing its intrinsic state (Gunderson 2000). Analogously, we can regard functional resilience as the amount of disturbance that a species assembly can absorb without changing its ecological function (Walker 1995). In this context, we would expect that functional redundancy is directly related to the resilience of an ecosystem function through buffering it from fluxes in species abundances and species interactions (Walker 1995; Gonzalez and Loreau 2009; Petchey and Gaston 2009b).

While it is recognized that the combined effects of species richness and the functional attributes of individual species on ecosystem function are complex (Reiss et al. 2009), it is difficult to fully incorporate and control for such complexity with empirical data (Meyer et al. 2009). This problem has caused a bias in research on the relationship between biodiversity and ecosystem functioning, with a disproportionate number of studies using empirical experimental approaches on simple systems and individual processes (Caliman et al. 2010). Yet, there is a need for assessing complexity and interactions of multiple factors for the design of appropriate empirical experiments and to refine current theory (Caliman et al. 2010; see also Peck 2004).

Previous work have investigated either the effects of the number of functional traits (Petchey and Gaston 2002) or of functional overlap (Tilman et al. 1997; Petchey 2000) on the relationship between functional diversity and species richness, and more recently, the effects of species richness and species composition on the resilience of functional diversity (Petchey and Gaston 2009b). However, to our knowledge, no one has yet simultaneously examined the combined effects of species richness, the number of functional traits, and the differences between species within these traits, i.e., the functional similarity between species, on species contributions to both functional diversity and redundancy. Here, we first review theoretical predictions regarding species contributions to functional diversity and redundancy, and then use simulated data sets to test the combined effects of species richness, number of functional traits, and species differences within traits on these contributions, as well as, on the overall functional diversity and redundancy of species assemblages. We use computer simulations instead of empirical data since it enables us to simultaneously test a fully factorial and balanced design. Throughout the remainder of this paper, we follow Petchey and Gaston (2002) and use FD to denote the total functional diversity of a species assembly or of individual species and analogously use the term FR to denote total functional redundancy. We use the lower case equivalents (fd and fr) to denote species contributions to diversity and redundancy.

\section{Theoretical effects of species richness and functional differences on functional diversity and redundancy}

Intuitively, the unique contribution to ecosystem function by any given species should consist only of those functional components that are not shared with other species (e.g., Walker 1992; Naeem 1998; Petchey et al. 2007). Although attempts have been made to incorporate the overall functional contributions of species' into a single quantified metric of functional diversity (Schmera et al. 2009), we suggest that functionally redundant components may be viewed as contributing to ecosystem function by improving an assembly's functional resilience by increasing its redundancy (Fig. 1a, e.g., Walker 1995; Naeem 1998). The proportion of a species' function that has a unique contribution to functional diversity should then decline with increased functional overlap, with a corresponding increase in the proportion that will contribute to redundancy (Fig. 1b). The unique contribution to functional diversity of a given species $\left(\mathrm{fd}_{i}\right)$ can then be described as its overall 
a

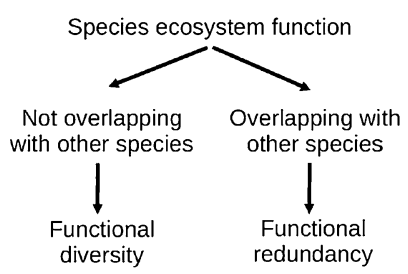

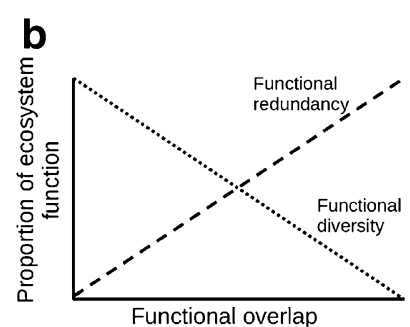

Fig. 1 a Schematic representation of the partitioning of a species' ecosystem function into unique contributions to functional diversity and to contribution to functional redundancy and $\mathbf{b}$ graphic presentation of how the proportion of species' ecosystem function that uniquely contribute to functional diversity versus redundancy varies over increasing levels of functional overlaps

ecosystem function $\left(\mathrm{fd}_{i}^{\prime}\right)$ minus its overlapping components. We can describe this for an assembly with $n$ species as:

$\mathrm{fd}_{i}=\mathrm{fd}_{i}^{\prime}-\sum_{j=1}^{n-1} \mathrm{fd}_{i}^{\prime} \cap \mathrm{fd}_{j}^{\prime}$

Following the same argument, we can describe a species' contribution to functional redundancy $\left(\mathrm{fr}_{i}\right)$ as the sum of all functional overlaps:

$\mathrm{fr}_{i}=\sum_{j=1}^{n-1} \mathrm{fd}_{i}^{\prime} \cap \mathrm{fd}_{j}^{\prime}$

This formulation highlights the complementary relationship between fd and fr following from our logic.

However, the similarity of overlapping components of a species (i.e., $\mathrm{fd}_{i}^{\prime} \cap \mathrm{fd}_{i}^{\prime}$ ) determines how its proportional contributions to functional diversity versus redundancy will vary with species richness (e.g., Petchey 2000). For instance, if all overlaps exist within the same components, there will be no relationship between species richness and the total proportion of a species' ecosystem function that overlaps with other species (Fig. 2a). Under this scenario, if more than one other species is present within an assembly, $\mathrm{fd}_{i}$ will not change with increasing species richness. This generates a linear increase in FD and FR with increasing species richness in any number of species above 1 (Fig. 2a). In contrast, if the overlapping components differ between species, the total overlap of a species will be related to species richness. In an extreme scenario, where each pair wise overlap is unique, there will be a linear relationship between species richness and the proportion of overlapping function, with a very rapid saturation in both $\mathrm{fd}_{i}$ and $\mathrm{FD}$ as species richness increases, especially with higher levels of pair wise overlaps (Fig. 2b). Since fr ${ }_{i}$, in Eqs. 1 and 2 are defined as the inverse of $\mathrm{fd}_{i}$, the inverse of these relationships applies to $\mathrm{fr}_{i}$ and FR (Fig 2a, b).

Although these predictions are intuitive, they highlight a few important points regarding the relationships between species contributions to functional diversity and redundancy, species richness, and functional similarities between species. First, they suggest that the similarity of pair wise overlaps in ecosystem function between species should be very influential on how $\mathrm{fd}_{i}$ and $\mathrm{fr}_{i}$ vary over different levels of species richness. Second, they suggest that the relative contributions to $\mathrm{fd}_{i}$ and $\mathrm{fr}_{i}$ should be complementary, so that an increase in the proportion of function that contributes to functional diversity should be coupled with a decrease in the proportion of function that contributes to functional redundancy. Third, since we can regard FD and FR as the cumulative functional contributions of species, they highlight that species assemblages with low levels of functional overlap should have high functional diversity but low functional redundancy, with the inverse situation for assemblages with high levels of functional overlap (e.g., Fig. 2a, b).

\section{Effects of species richness, number of functional traits, and species differences on functional diversity and resilience}

Simulation methods and analyses

We constructed simulated species assemblages ranging from 5 to 50 species (in increments of $5,10,25$, and 50 species), and sequentially varied the number of functional traits to $1,5,10$, and 20 for each level of species richness. Within each species richness-trait combination, we drew the trait values for each trait independently from uniform distributions. We simulated species differences within traits by varying the range of these distributions, so that a distribution with a large range would, on average, generate a data set with less similar species than data drawn from a distribution with a narrower range. All traits of a species assembly were drawn from distributions with equal range. We used uniform distributions since we were interested in using the range of the distributions to simulate assemblages with different relative differences between species, and values drawn from uniform distributions will be more evenly spaced throughout the defined variable space compared with values drawn from distributions defined by a measure of central tendency. We let the distributions vary from 0.1 to 100 , in increments of $0.1,1,5,10,25,50$, and 100 , and repeated the random draws of trait values ten times for each combination of species, number of traits, and trait ranges.

Following Petchey and Gaston (2002), we conducted a cluster analyses on each simulated assembly and estimated the total FD as the sum of all the branch lengths within each dendrogram and $\mathrm{fd}$ as the average unique branch length of all species in a simulated assembly. We estimated the overall FR 
a

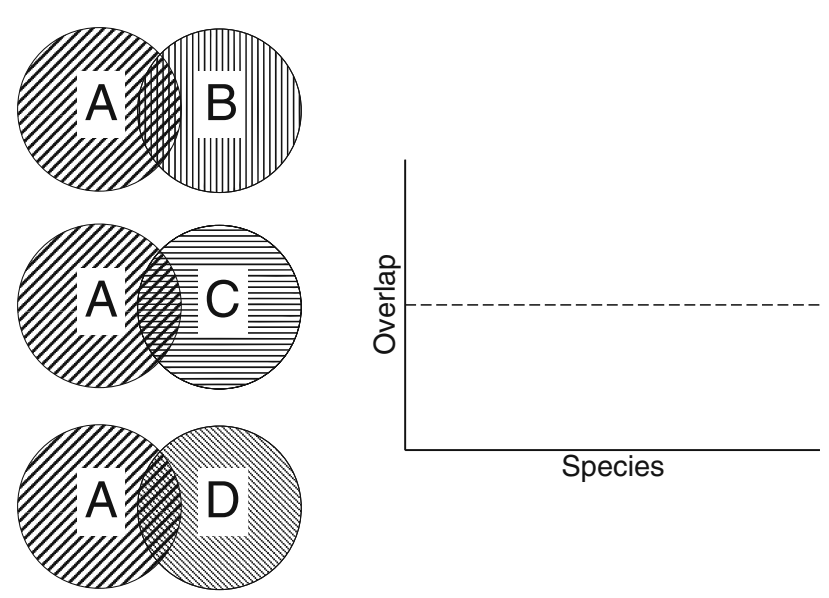

b
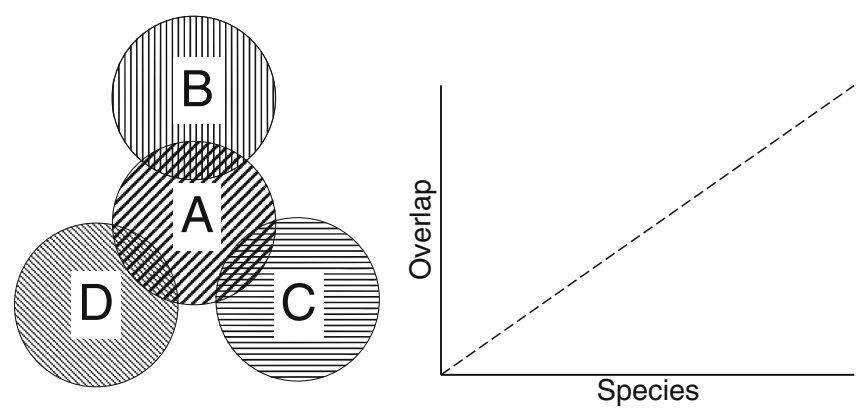
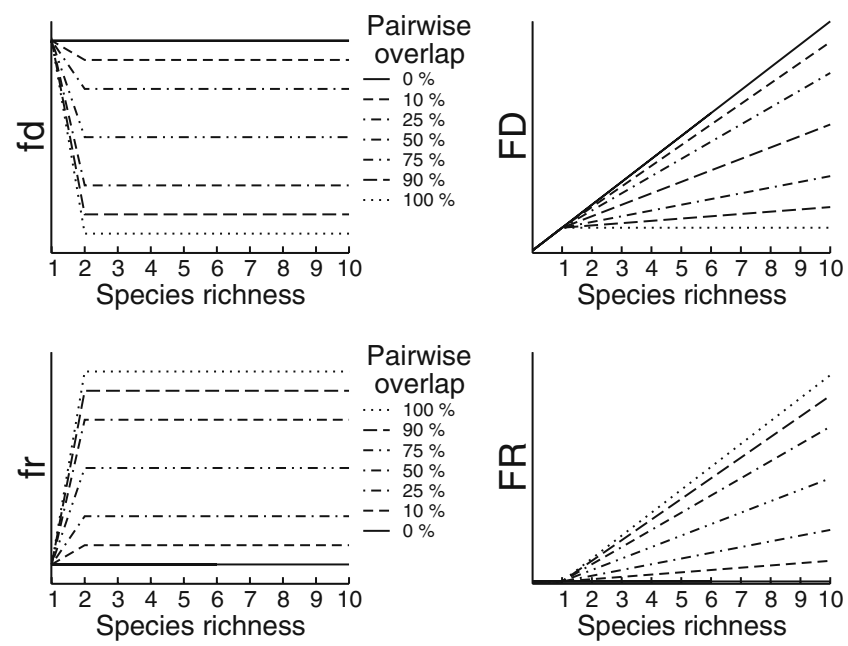
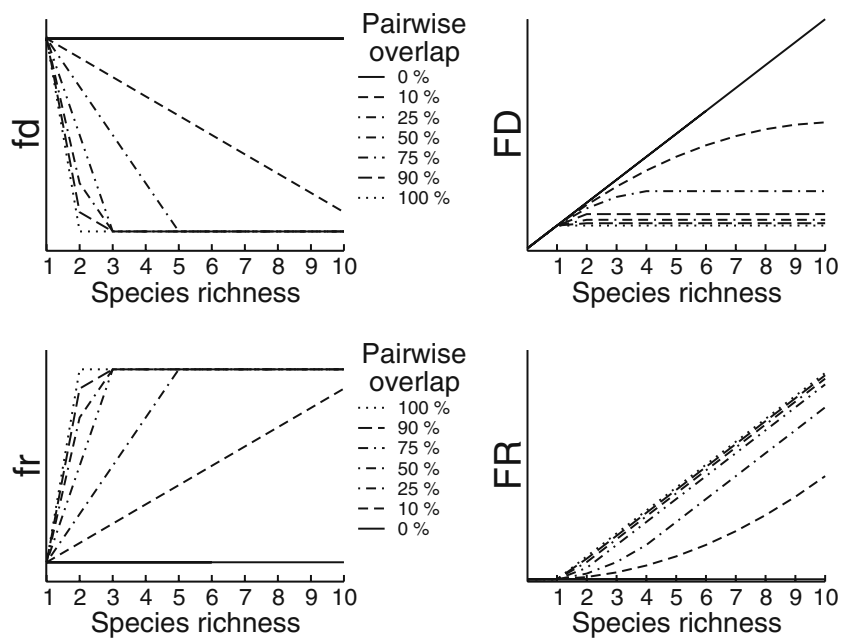

degree of pair wise functional overlaps a if the overlapping components are identical across all species pairs for a given species and $\mathbf{b}$ the overlapping components are unique across all species pairs for a given species

by Petchey and Gaston (2009a), in which subsets of a regional assemblage are compared to the full assemblage. However, we believe that our approach is more relevant for our simulations since it highlights the relative effects of functional similarity between species while still avoiding the methodological problems of using unscaled values (e.g., Podani and Schmera 2006). We scaled FR to represent the proportion of redundancy in each tree in relation to its total branch length and fr to represent the average proportion of redundancy in each species contribution (i.e., the total species contribution with the unique functional contribution subtracted). Details for the calculations and scaling for a sample tree are exemplified in Fig. 3.

We used Euclidean distance matrices since our data set contained the same type of data, and the unweighted pairgroup method using arithmetic averages (UPGMA) clustering method (Petchey and Gaston 2002). Since all trait values within each simulated assembly were drawn from 


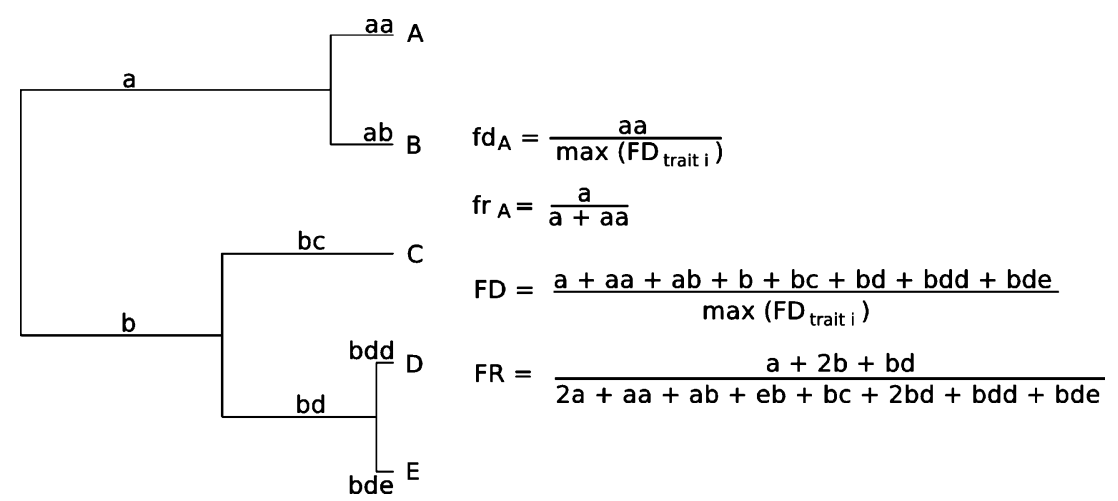

Fig. 3 Visualization of a simulated cluster tree containing five species $(a-e)$, and corresponding formulas for how the scaled functional metrics fd, fr, FD, and FR were calculated. Individual contribution to functional diversity fd and total functional diversity FD were scaled after the maximum FD value for each run of simulations with each

the same family of underlying distributions, we calculated the distance matrices from unscaled data tables.

We constructed generalized linear models with a gamma error distribution using fd, fr, FD, and FR from our simulated data as response variables, and complete designs containing species richness, trait richness, and species differences as well as all interactions as predictors. We fitted reciprocal models with inverse link functions since these provided better fit to the data than models using identity links for all response variables based on corresponding AIC values. These models represents a linearization of the asymptotic Michaelis-Menten curve (Crawley 2002) and were tested against models using linear identity links due to the expected asymptotic relationships between functional diversity metrics and the predictors (e.g., Petchey and Gaston 2002). We have not reported probabilities of statistical inference from these models, since such values are based on sample size, which in our case was arbitrarily determined (e.g., Dalerum et al. 2008), but instead evaluated the relative influence of each term based on its standardized coefficient (i.e., the absolute values of raw coefficients divided by their standard error, Selvin 1998). All simulations and analyses were conducted using the statistical package $\mathrm{R}$ version 2.11.1 for Linux (http://www.r-project.org).

\section{Simulation results}

Species difference were the most influential term on $\mathrm{fd}$ followed by an interaction between species differences, number of traits, and species richness (Table 1), in which the interactions between species differences and species richness was accentuated in assemblages with only a single functional trait (Fig. 4a-c). In contrast, variation in fr was mostly influenced by the number of functional traits and species richness (Table 1). There was a higher proportion of function contributing to redundancy in assemblages with a number of traits, whereas individual contribution of redundancy fr was scaled as the average proportion of each species' total branch length that were non-unique and overall functional redundancy FR as the proportion of the sum of all branch lengths within a tree that were non-unique

low number of traits and a positive relationship between $\mathrm{fr}$ and species richness (Fig. 4d-f), and this relationship was not altered substantially by either the number of traits or species differences within these traits (Table 1).

Species richness and species differences interacted strongly in their effects on FD (Table 1), with increasing effects of species richness in assemblages with large differences between species and increasing effects of species differences in assemblages with a large number of species (Fig $5 \mathrm{a}-\mathrm{c}$ ). This interaction was more pronounced in assemblages with a large number of traits. The most influential terms for variation in FR was species richness and number of traits (Table 1), with increasing redundancy in species rich assemblages and in assemblages with a low number of traits (Fig $5 d-f)$.

\section{Discussion}

Our simulations highlighted that species richness, number of traits, and species differences within traits can interact in their effects on both functional diversity and redundancy. Such complex interactions between functional attributes among species and their relative contributions to ecosystem function support recent suggestions that an approach focusing on multiple processes and ecological interactions may be necessary to fully understand the relationship between biological diversity and ecosystem processes (Balvanera et al. 2006; Duffy et al. 2007; Reiss et al. 2009; Caliman et al. 2010).

Some interesting patterns emerged from our simulations. First, functional diversity and redundancy did not appear to be complementary over varying degrees of functional similarity between species. This was true both for species contributions ( $\mathrm{fd}$ and $\mathrm{fr}$ ) as well as the overall values for 
Table 1 Raw and standardized regression coefficients for main and interaction effects of number of species, number of traits, and species differences on individual contribution to functional diversity (fd) and proportion of redundancy in total species function (fr) as well as on total functional diversity (FD) and redundancy (FR) in simulated species assemblages

\begin{tabular}{|c|c|c|}
\hline Predictor & $\beta$ coefficent & Standardized $\beta$ coefficent \\
\hline \multicolumn{3}{|l|}{$\mathrm{fd}$} \\
\hline Species differences $^{\mathrm{a}}$ & 514 & 36.0 \\
\hline Species differences $\times$ Number of traits $^{\mathrm{b}} \times$ Species richness $^{\mathrm{c}}$ & $-5,430$ & 20.9 \\
\hline Species differences $\times$ Number of traits & 627 & 15.0 \\
\hline Species differences $\times$ Species richness & $-1,010$ & 10.7 \\
\hline Number of traits & 14.6 & 1.42 \\
\hline Number of traits $\times$ Species richness & -75.5 & 1.33 \\
\hline Species richness & -7.15 & 0.28 \\
\hline \multicolumn{3}{|l|}{ fr } \\
\hline Number of traits & -1.21 & 19.83 \\
\hline Species richness & 11.2 & 19.2 \\
\hline Number of traits $\times$ Species richness & -9.17 & 13.3 \\
\hline Species differences & 0.007 & 0.71 \\
\hline Species differences $\times$ Species richness & -0.07 & 0.70 \\
\hline Species differences $\times$ Number of traits & -0.007 & 0.50 \\
\hline Species differences $\times$ Number of traits $\times$ Species richness & 0.05 & 0.42 \\
\hline \multicolumn{3}{|l|}{ FD } \\
\hline Species differences $\times$ Species richness & 2.87 & 99.1 \\
\hline Species differences $\times$ Number of traits $\times$ Species richness & -263 & 76.8 \\
\hline Species differences & 49.7 & 35.4 \\
\hline Species differences $\times$ Number of traits & 54.3 & 24.0 \\
\hline Species richness & 2.28 & 2.71 \\
\hline Number of traits $\times$ Species richness & -1.89 & 1.92 \\
\hline Number of traits & 0.04 & 0.84 \\
\hline \multicolumn{3}{|l|}{ FR } \\
\hline Species richness & 23.7 & 25.7 \\
\hline Number of traits & -1.64 & 20.3 \\
\hline Number of traits $\times$ Species richness & -17.5 & 16.4 \\
\hline Species differences & 0.01 & 0.90 \\
\hline Species differences $\times$ Species richness & -0.12 & 0.75 \\
\hline Species differences $\times$ Number of traits & -0.01 & 0.73 \\
\hline Species differences $\times$ Number of traits $\times$ Species richness & 0.09 & 0.49 \\
\hline
\end{tabular}

Data were quantified from dendrograms constructed using cluster analyses following Petchey and Gaston (2002). Results are from reciprocal generalized linear models with inverse link function and gamma error constructed on the output from simulations where number of species was set to $5,10,25$, and 50 and number of traits to $1,5,10$, and 20 . Trait values were drawn from uniform distributions and species differences were altered by changing the range of these distributions. Each combination of species richness, number of traits, and species differences was repeated ten times. Terms are ordered by the standardized coefficients, i.e., the absolute values of the raw coefficients divided by their standard errors

${ }^{a}$ Species differences were quantified as the mean range of trait values calculated across species for each trait

${ }^{\mathrm{b}}$ Number of functional traits in each simulated assemblage

${ }^{\mathrm{c}}$ Number of species present in assembly

simulated assemblages (FD and FR). This contradicts that a high degree of functional similarity should generate a high degree of redundancy but a relatively lower diversity. Instead, our results suggest that while both fd and FD increase with increasing differences between species, as predicted, both fr and FR appear to be unaffected by such differences. Instead, redundancy seems to largely be determined by the main effects of the number of separating traits and species richness. These results generally suggest that functional diversity and redundancy may not be completely complementary and that functional similarities between species may not directly influence functional redundancy. 
Fig. 4 Effects of species richness and species differences on the $\mathbf{a}-\mathbf{d}$ average unique species contribution to functional diversity fd and $\mathbf{e}-\mathbf{h}$ the average proportion of redundancy in total species function fr over four dimensions of separating trait space (number of functional traits). Data were quantified from dendrograms constructed using cluster analyses, and the surface planes were parameterized from reciprocal generalized linear models based on data generated by simulations where number of species was set to 5 , 10,25 , and 50 and number of traits to $1,5,10$, and 20. From the simulated trees, we calculated $\mathrm{fd}$ as the average unique branch lengths for all species in a simulated assembly, scaled by the maximum value of total functional diversity FD for each simulation run within the same number of traits (Petchey and Gaston 2002), and fr as the average of the non-unique branch length divided by the total branch length for each species a

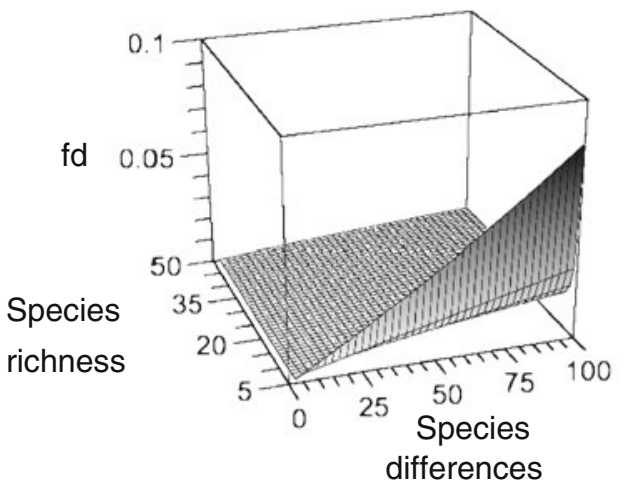

b

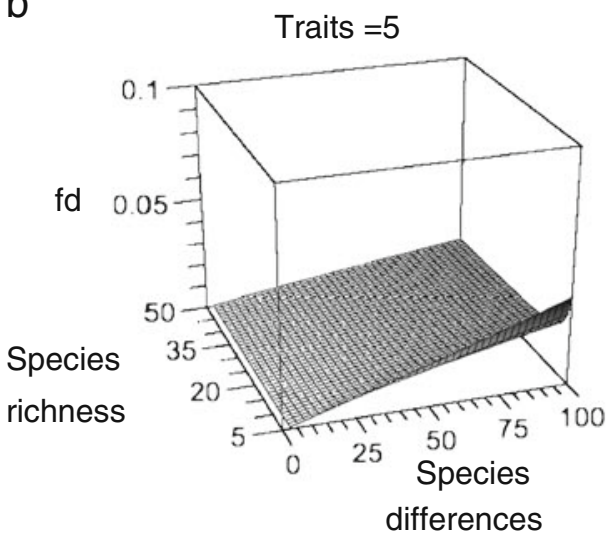

C

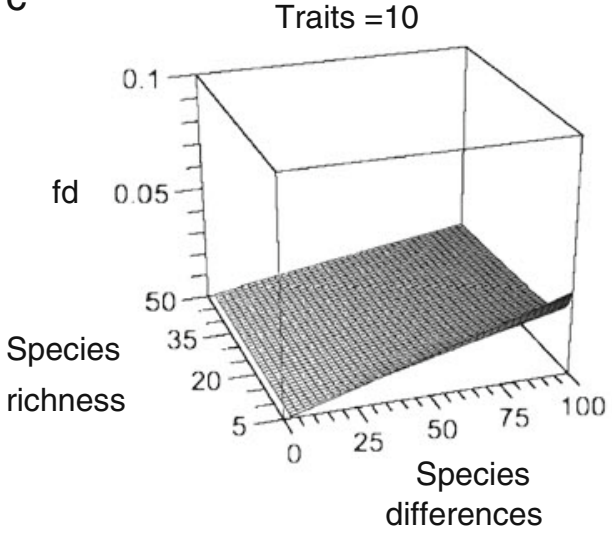

d

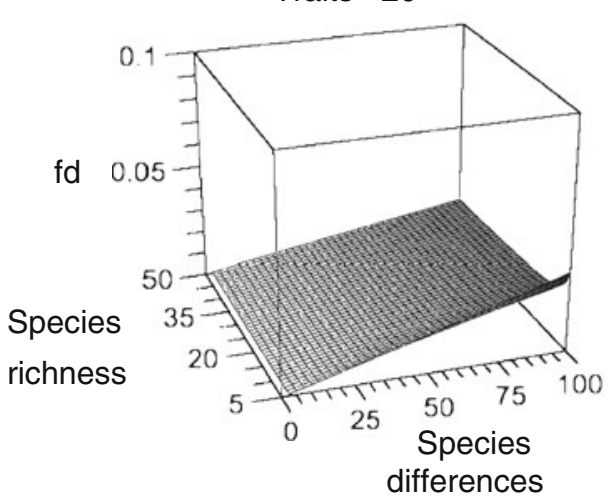

e

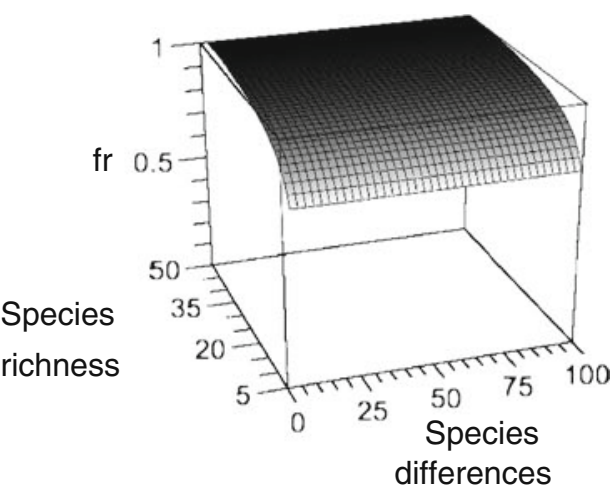

f

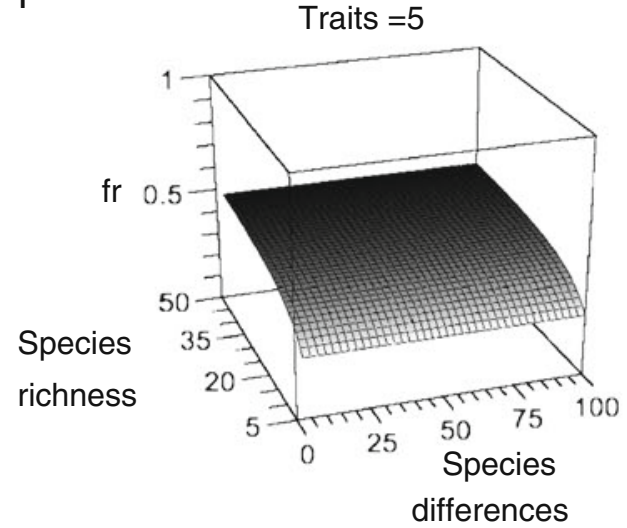

g

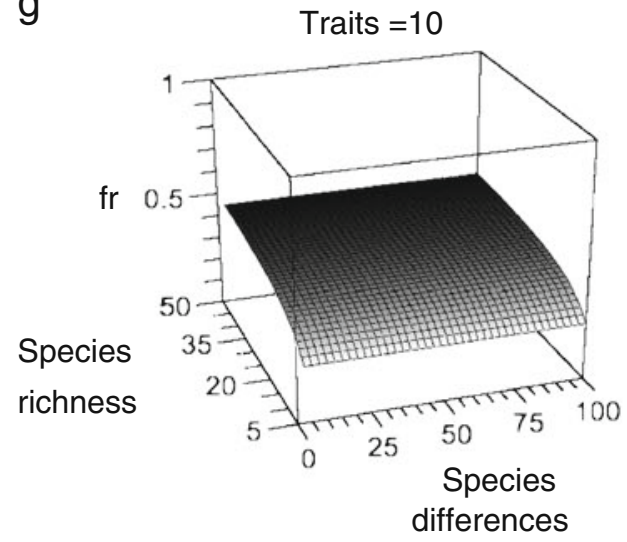

h

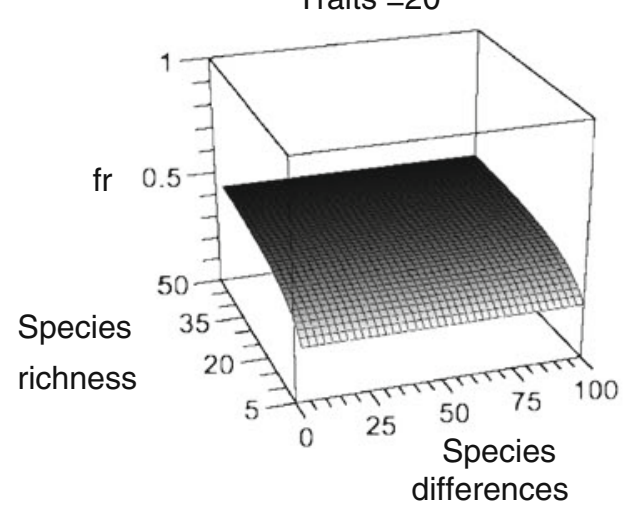


Fig. 5 Effects of species richness and species differences on overall a-c functional diversity FD and $\mathbf{d}-\mathbf{f}$ redundancy FR in simulated species assemblages over four dimensions of separating trait space (number of functional traits). Data were quantified from dendrograms constructed using cluster analyses, and the surface planes were parameterized from reciprocal generalized linear models based on data generated by simulations where number of species was set to $5,10,25$, and 50 and number of traits to $1,5,10$, and 20 . From the simulated trees, we quantified FD as the total branch length of each tree scaled by the maximum value of FD for each simulation run within the same number of traits (Petchey and Gaston 2002) and FR as the proportion of non-unique branch lengths within each tree a

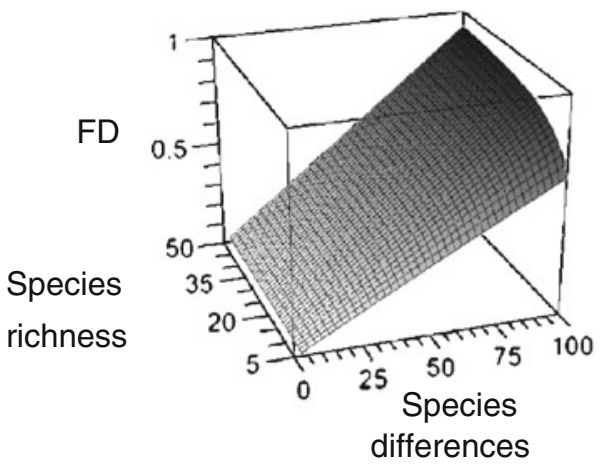

b

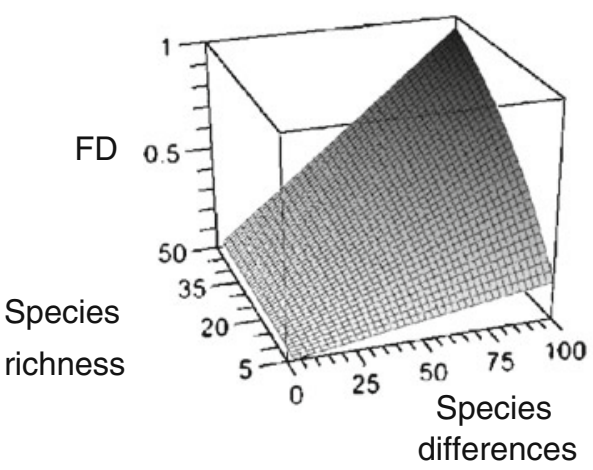

C

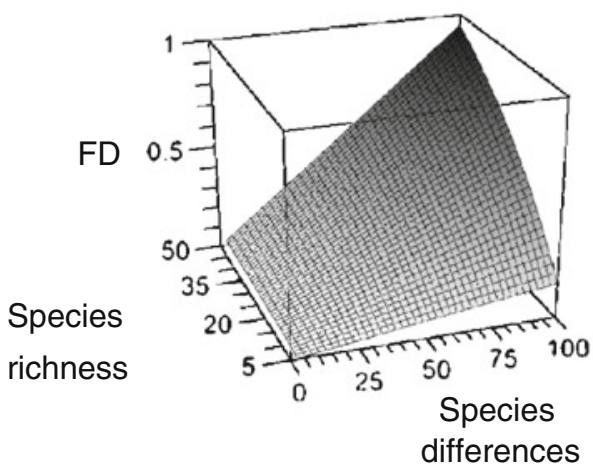

d

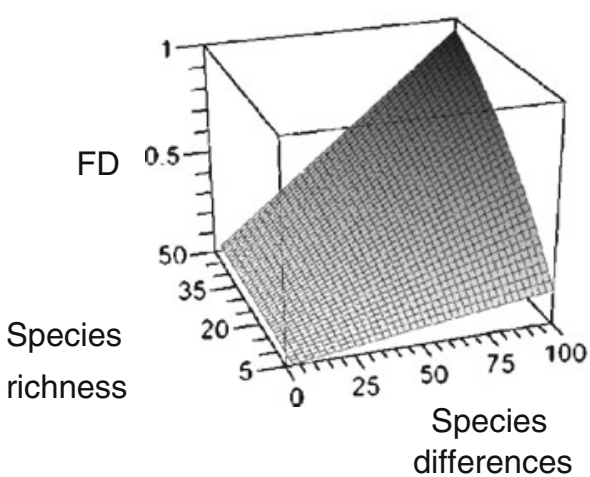

e Traits $=1$

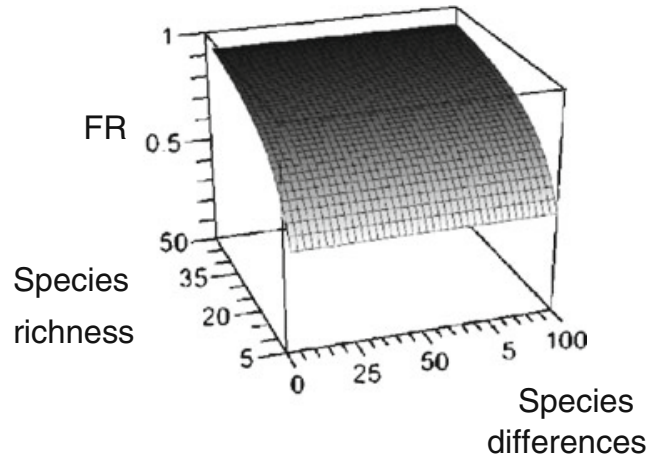

f

Traits $=5$

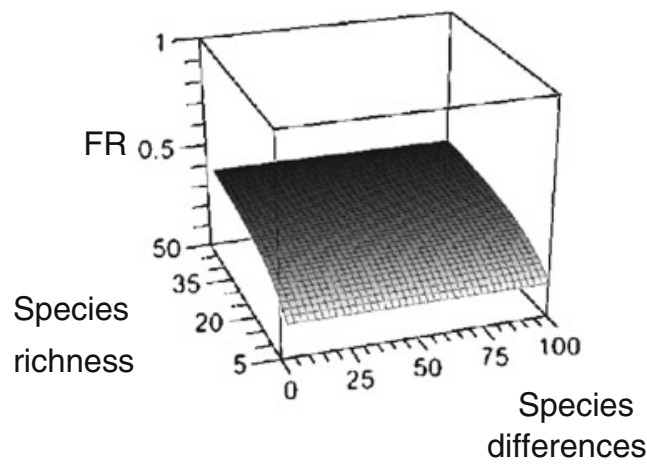

g

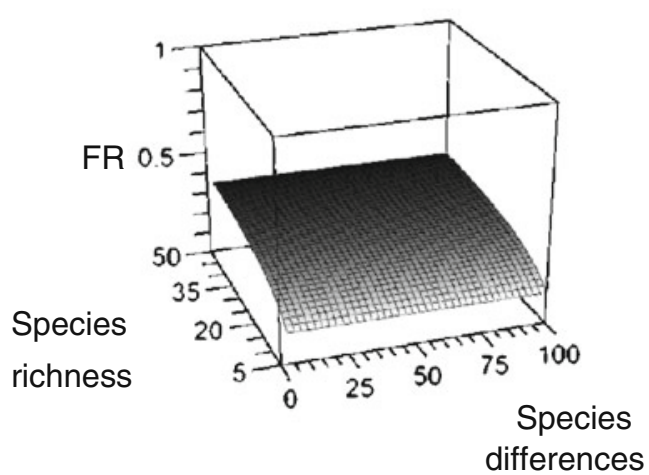

h

Traits $=20$
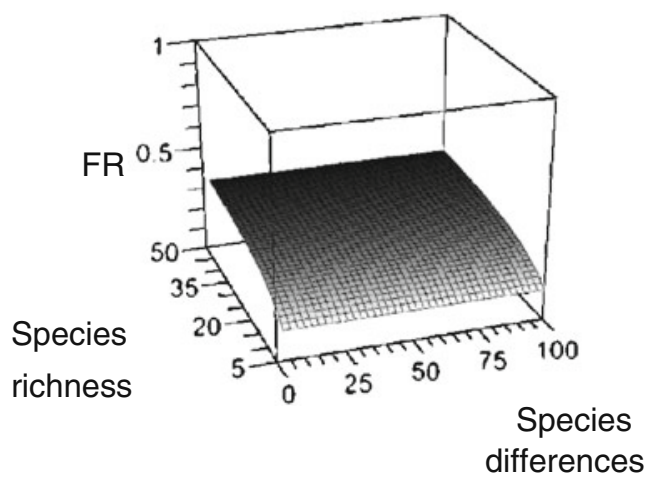
Secondly, our results suggest that the number of separating traits was the most influential term for functional redundancy, but that redundancy was higher in assemblages with few functional traits. These results contradict the assumption that increasing complexity would add increasing stability (e.g., Bersier 2007), since we would intuitively regard a species assembly separated by a multidimensional trait space to be more complex than an assembly separated by a single or only a few function traits. The results also contradict the assumption that species differences should be highly influential on redundancy. However, Petchey and Gaston (2002) acknowledged that in assemblages with a high dimensionality of the trait space, species pairs tend to differ similarly, whereas in assemblages with a low dimensionality of the trait space, some species pairs tend to be similar while others tend to be different. Although this pattern may explain the effect of number of traits on functional redundancy found in our study, it does not readily explain the observed lack of an effect of species similarity. We therefore suggest that further work is directed towards exploring the specific relationships between species attributes and the proportion of redundancy within each species as well as how species attributes affect functional redundancy in complete assemblages.

Thirdly, our results suggest that the relative effects of species richness and functional attributes among species affect individual contributions differently compared with the overall functional diversity of complete assemblages. This may seem paradoxical, since the value of an assembly is the cumulative contributions of the species which it consists of. However, because of the cumulative nature of assembly level metrics, even effects that are relatively minor for individual contributions could be magnified at the assembly level, especially in species rich assemblages. This scale dependence suggest that one cannot generalize effects of species richness and species function on the assembly level into similar effects on individual contributions, and vice versa, and highlight that metrics of functional diversity should simultaneously be quantified at both levels to fully capture the effects of species richness and species function on the functional structure of species communities.

Finally, we note that there is a general scarcity of studies that have tested these relationships empirically. Although species richness has been positively related to both functional diversity and resilience (e.g., Naeem et al. 1994; Tilman 1999; Balvanera et al. 2006; Fischer et al. 2007; Petchey et al. 2007), studies that have measured species specific contributions are absent in the literature, with a few exceptions (e.g., Balvanera et al. 2005; Baker et al. 2009; Luck et al. 2009; Schmera et al. 2009). We see a similar pattern for studies evaluating the effects of functional attributes among species on their contribution to ecosystem function and stability, although functional attributes among species recently have been related to the overall functional diversity of empirical assemblages (Dang et al. 2005; Micheli and Halpern 2005; McKie et al. 2008; Petchey et al. 2007). This highlights that there is still a gap in knowledge regarding the empirical mechanisms with which each species interactively contribute to ecosystem function and stability. Our results suggest that such data are necessary to fully understand the role of species in ecosystems, and hence be able to predict the functional effects of alterations in species compositions on different scales.

In conclusion, our simulations highlighted that species richness and functional attributes among species can interact in their effects on functional diversity and redundancy, and that these interactions may be more pronounced for the overall functional diversity of assemblages compared to species contributions. Our simulations further suggested that functional similarity among species have little effects on the functional redundancy of species assemblages, as well as, on species contributions to redundancy. Finally, our simulations suggested scale dependence in the relative effects of species richness and functional attributes of species, so that the relative influence of these factors may affect individual contributions differently compared to the overall ecosystem function of species assemblages. Based on these results, we stress that studies on the relationship between biological diversity and ecosystem processes will benefit from focusing on multiple processes and ecological interactions, and that the relative functional attributes among species will have pivotal roles for the ecosystem function of a given species assembly.

Acknowledgments This research was made possible by a postdoctoral fellowship from the National Research Foundation of South Africa, a senior research fellowship from the University of Pretoria, and a grant from the National Geographic Society/Waits foundation. Lydia Belton, Andre Ganswindt, Maria Miranda Garcia-Roves, and two anonymous reviewers gave valuable comments on the manuscript.

\section{References}

Baker TR, Phillips OL, Laurance WF, Pitman NCA, Almeida S, Arroyo L, DiFiore A, Erwin T, Higuchi N, Killeen TJ, Laurance SG, Nascimento H, Monteagudo A, Neill DA, Silva JNM, Malhil Y, pez Gonzalez GL, Peacock J, Quesada CA, Lewis SL, Lloyd J (2009) Do species traits determine patterns of wood production in Amazonian forests? Biogeosci 6:297-307

Balvanera P, Kremen C, Matrinez-Ramos M (2005) Applying community structure analysis to ecosystem function: examples from pollination and carbon storage. Ecol Appl 15:360-375. doi:10.1890/03-5192 
Balvanera P, Pfisterer AB, Buchmann N, He J-S, Nakashizuka T, Raffaelli D, Schmid B (2006) Quantifying the evidence for an effect of biodiversity on ecosystem functioning and services. Ecol Lett 9:1146-1156. doi:10.1111/j.1461-0248.2006.00963.x

Bersier LF (2007) A history of the study of ecological network. In: Képès $F$ (ed) Biological networks. Complex systems and interdisciplinary science, vol. 3. World Scientific, pp 365 421

Bracken MES, Friberg SE, Gonzalez-Dorantes CA, Williams SL (2009) Functional consequences of realistic biodiversity changes in a marine ecosystem. Proc Natl Acad Sci USA 105:924-928. doi:10.1073/pnas.0704103105

Caliman A, Pires AF, Esteves FA, Bozelli RL, Farjalla VF (2010) The prominence of and biases in biodiversity and ecosystem functioning research. Biodiv Cons 19:651-664. doi:10.1007/s10531-009-9725-0

Crawley MJ (2002) Statistical computing an introduction to data analysis using s-plus. Wiley, Chichester

Dalerum F, Shults B, Kunkel K (2008) Estimating relative importance of sustainable harvest in wolverine (Gulo gulo) populations using logistic regression. J Wildl Manage 72:1125-1132. doi:10.2193/ 2007-336

Dalerum F, Cameron EZ, Kunkel KE, Somers MJ (2009) Continental patterns of carnivore guild depletions: implications for prioritizing global carnivore conservation. Biol Lett 5:35-38. doi:10.1098/rsbl.2008.0520

Dang CK, Chauvet E, Gessner MO (2005) Magnitude and variability of process rates in fungal diversity-litter decomposition relationships. Ecol Lett 8:1129-1137. doi:10.1111/j.1461-0248.2005.00815.x

Duffy JE, Carnidale BJ, France KE, McIntryre PB, Thebault E, Loreau M (2007) The functional role of biodiversity in ecosystems: incorporating trophic complexity. Ecol Lett 10:522-538. doi:10.1111/j.1461-0248.2007.01037.x

Fischer J, Lindenmayer DB, Blomberg SP, Montague-Drake M, Felton A, Stein JA (2007) Functional richness and relative resilience of bird communities in regions with different land use intensities. Ecosystems 10:964-974. doi:10.1007/s10021-007-9071-6

Fukami T, Bezemer TM, Mortimer SR, Van der Putten WH (2005) Species divergence and trait convergence in experimental plant community assemblage. Ecol Lett 8:1238-1290. doi:10.1111/ j.1461-0248.2005.00829.x

Griffin JN, Mendez V, Johnson AF, Jenkins SR, Foggo A (2009) Functional diversity predicts overyielding effects of species combinations on primary productivity. Oikos 118:37-44. doi:10.1111/j.1600-0706.2008.16960.x

Gonzalez A, Loreau M (2009) The causes and consequences of compensatory dynamics in ecological communities. Ann Rev Ecol Syst Evol 40:393-414. doi:101146/annurev.ecolsys.39.110707.173349

Gunderson LA (2000) Ecological resilience - in theory and application. Ann Rev Ecol Syst 31:425-439

Hector A, Joshi J, Lawler SP, Spehn EM, Wilby A (2001) Conservation implications for the link between biodiversity and ecosystem functioning. Oecologia 129:624-628. doi:10.1007/ s004420100759

Heino J (2005) Functional biodiversity of macroinvertebrate assemblages along major ecological gradients of boreal headwater streams. Freshw Biol 50:1578-1587. doi:10.1111/j.1365-2427.2005.01418.x

Holling CS (1973) Resilience and stability of ecological systems. Ann Rev Ecol Syst 4:1-23

Johnson KH, Vogt KA, Clark HJ, Schmitz OJ, Vogt DJ (1996) Biodiversity and the productivity and stability of ecosysems. Trends Ecol Evol 11:372-377. doi:10.1016/0169-5347(96)10040-9

Lawton JH, Brown VK (1993) Redundancy in ecosystems. In: Schultze ED, Mooney DA (eds) Biodiversity and ecosystem function. Springer, pp 255-270

Luck GW, Harrington R, Harrison PA, Kremen C, Berry PM, Bugter R, Dawson TR, De Bello F, Diaz S, Feld CK, Haslett
JH, Hering D, Kontogianni A, Lavorel S, Rounsevell M, Samways MJ, Sandin L, Settele J, Sykes MT, Van Den Hove S, Vandewalle M, Zobel M (2009) Quantifying the contribution of organisms to the provision of ecosystem services. Bioscience 59:223-235

Loreau M (2004) Does ecological redundancy exist? Oikos 103:606611. doi:10.1025/bio.2009.59.3.7

Loreau M, Naeem S, Inchausti P, Bengtson J, Grime JP, Hector A, Hooper DU, Huston MA, Rafaelli D, Schmid B, Tilman D, Wardle DA (2001) Biodiversity and ecosystem functioning: current knowledge and future challenges. Science 294:804-808. doi:10.1126/science.1064088

McKie BG, Woodward G, Hladyz S, Nistorescu M, Preda E, Popescu C, Giller PS, Malmqvist B (2008) Ecosystem functioning in stream assemblages from different regions: contrasting responses to variation in detritivore richness, evenness and density. J Anim Ecol 77:495-504. doi:10.1111/ j.1365-2656.2008.01357.x

Meyer KM, Mooij WM, Vos M, Hol WHG, Van der Putten VH (2009) The power of simulating experiments. Ecol Mod 220:2594-2597. doi:10.1016/j.ecolmodel.2009.06.001

Micheli F, Halpern BS (2005) Low functional redundancy in coastal marine assemblages. Ecol Lett 8:391-400. doi:10.1111/j.14610248.2005.00731.x

Naeem S (1998) Species redundancy and ecosystem reliability. Cons Biol 12:39-45. doi:10.1046/j.1523-1739.1998.96379.x

Naeem S (2002) Ecosystem consequences of biodiversity loss: the evolution of a paradigm. Ecology 83:1537-1552. doi:10.1890/ 0012-9658(2002)083[1537:ECOBLT]2.0.CO;2

Naeem S, Wright JP (2003) Disentangling biodiversity effects on ecosystem functioning: deriving solutions to a seemingly insurmountable problem. Ecol Lett 6:567-579. doi:10.1046/ j.1461-0248.2003.00471.x

Naeem S, Thomson LJ, Lawler SP, Lawton JH, Woodfin RM (1994) Declining biodiversity can alter the performance of ecosystems. Nature 368:734-737. doi:10.1038/368734a0

Peck SL (2004) Simulation as experiment: a philosophical reassessment of biological modelling. Trends Ecol Evol 19:530-534. doi:10.1016/j.tree.2004.07.019

Petchey OL (2000) Species diversity, species extinction, and ecosystem function. Am Nat 155:696-702. doi:10.1086/303352

Petchey OL, Gaston KJ (2002) Functional diversity (FD), species richness and community composition. Ecol Lett 5:402-411. doi:10.1046/j.1461-0248.2002.00339.x

Petchey OL, Gaston KJ (2006) Functional diversity: back to basics and looking forward. Ecol Lett 9:741-758. doi:10.1111/j.14610248.2006.00924.x

Petchey OL, Gaston KJ (2009a) Dendrograms and measuring functional diversity: a second instalment. Oikos 118:1118-1120. doi:10.1111/j.1600-0706.2009.17403.x

Petchey OL, Gaston KJ (2009b) Effects on ecosystem resilience of biodiversity, extinctions, and the structure of regional species pools. Theor Ecol 2:177-187. doi:10.1007/s12080009-0041-9

Petchey OL, Evans KL, Fishburn IS, Gaston KJ (2007) Low functional diversity and no redundancy in British avian assemblages. J Anim Ecol 76:977-985. doi:10.1111/j.1365-2656.2007.01271.x

Podani J, Schmera D (2006) On dendrogram measures of functional diversity. Oikos 115:179-185. doi:10.1111/j.2006.00301299.15048.x

Reiss J, Bridle JR, Montoya JM, Woodward G (2009) Emerging horizons in biodiversity and ecosystem functioning research. Trends Ecol Evol 24:505-514. doi:10.1016/j.tree.2009.03.018

Rodrigues ASL, Gaston KJ (2002) Maximizing phylogenetic diversity in the selection of networks of conservation areas. Biol Conserve 105:103-111. doi:10.1016/S0006-3207(01)00208-7 
Schmera D, Podani J, Eros T (2009) Measuring the contribution of community members to functional diversity. Oikos 118:961-971. doi:10.1111/j.1600-0706.2009.17076.x

Schwarz MW, Brigham CA, Hoeksema JD, Lyon KG, Mills MH, van Mantgem PJ (2000) Linking biodiversity to ecosystem function: implications for conservation biology. Oeologia 122:297-305. doi:10.1007/s004420050035

Selvin S (1998) Modern applied biostatistical methods. Oxford University Press, Oxford

Srivistava DS, Vellend M (2005) Biodiversity-ecosystem function research: is it relevant to conservation. Ann Rev Ecol Evol Syst 36:267-294. doi:10.1146/annurev.ecolsys.36.102003.152636
Tilman D (1999) Ecology — diversity in European grasslands. Science 286:1099-1100. doi:10.1126/science.277.5330.1300

Tilman D (2001) Functional diversity. In: Levin SA (ed) Encyclopaedia of biodiversity. Academic, San Diego, p 943

Tilman D, Lehman CL, Thomson KT (1997) Plant diversity and ecosystem productivity: theoretical considerations. Proc Natl Acad Sci USA 94:1857-1861

Walker B (1992) Biodiversity and ecological redundancy. Conserve Biol 6:18-23. doi:10.1046/j.1523-1739.1992.610018.x

Walker B (1995) Conserving biological diversity through ecosystem resilience. Conserve Biol 9:747-752. doi:10.1046/j.15231739.1995.09040747.x 